\title{
ANÁLISE SITUACIONAL DA IMPLANTAÇÃO DO BIM COMO APOIO AO PROCESSO DE PROJETO: ESTUDO DE CASO NA CIDADE DE MACEIÓ-AL1
}

\author{
SITUATION ANALYSIS OF THE BIM IMPLEMENTATION AS SUPPORT FOR \\ THE PROJECT PROCESS: A CASE STUDY IN MACEIÓ -AL CITY
}

\author{
Tiago de Vasconcelos Gonçalves Ferreira \\ Universidade Federal de Alagoas \\ tvasconcelosgf@gmail.com \\ Adriana de Oliveira Santos Weber \\ Universidade Federal de Alagoas \\ os.adriana@gmail.com
}

\begin{abstract}
Resumo
Com o surgimento do Building Information Modeling, o futuro do trabalho colaborativo será definido através do uso de um modelo único integrado, aos quais todos os agentes envolvidos poderão acessar e inserir as informações de suas respectivas disciplinas em um modelo de informações compartilhadas. Para que seja possível esse avanço na nova forma de projetar utilizando os conceitos do processo de projeto com o BIM, são necessários alguns esforços individuais, relativos ao uso e operação da tecnologia, e esforços coletivos, exigindo cooperação e a colaboração entre os intervenientes. Este trabalho buscou avaliar, através da metodologia de pesquisa Survey, o panorama do processo de projeto estabelecido em Maceió-AL e quais os condicionantes que são favoráveis para o uso do BIM. A pesquisa mostrou que as tecnologias utilizadas em obra para colaboração não trouxeram mudanças reais na forma de condução do processo de projeto, além da não existência de um coordenador com atuação mais incisiva nas várias etapas do processo. O termo "BIM" mostrou-se bastante difundido, apesar de alguns equívocos em sua definição. Dessa forma, as propostas estudadas para implantação do BIM apontam para uma modelagem isolada e pouco colaborativa que gera dúvidas das reais melhorias que trará ao processo de projeto.
\end{abstract}

Palavras-chave: Modelagem da Informação da Construção. Tecnologia da Informação. Coordenação e Colaboração em Projetos

\begin{abstract}
With the emergence of building information modeling, the future of collaborative work will be defined through the use of a single integrated model, to which all stakeholders can access and enter information of their respective disciplines in a model of shared information. To be able to advance in this new form of design using the concepts of the design process with the BIM, it takes a few individual efforts, concerning the use and operation of technology, and collective efforts, requiring cooperation and collaboration among stakeholders. This study evaluated by Survey research methodology, the panorama of the design process established in Maceió-AL and what conditions that are favorable for the use of BIM. Research has shown that the technologies used on site for collaboration did not bring real changes in the conduction of the design process, besides the absence of a coordinator with more incisive role in the various stages of the process. The term "BIM" proved to be quite widespread, although some misconceptions in its definition. Thus, the proposals studied for BIM deployment link
\end{abstract}

\footnotetext{
${ }^{1}$ FERREIRA, T. V. G.; SANTOS, A. O. W. Análise situacional da implantação do BIM como apoio ao processo de projeto: estudo de caso em Maceió-AL. In: ENCONTRO BRASILEIRO DE TECNOLOGIA DE INFORMAÇÃO E COMUNICAÇÃO NA CONSTRUÇÃO, 7., 2015, Recife. Anais... Porto Alegre: ANTAC, 2015.
} 
to a secluded and little collaborative modeling that generates doubts of the real improvements that will bring to the design process.

Keywords: Building Information Modeling. Information Technology. Coordination and Collaboration Projects

\section{INTRODUÇÃO}

A construção civil é um setor caracterizado pelo alto índice de desperdício, que varia entre $5 \%$ e $8 \%$ do total da obra (AGOPYAN, 2001). Diversos trabalhos mostram que grande parte dessas perdas são provenientes da baixa qualidade dos projetos que são entregues nas obras que chegam com erros e incoerências que obrigam os responsáveis pela execução a tomar decisões dentro do próprio canteiro de obras a fim de solucionar os problemas, aumentando o custo com serviços não previstos em orçamento e também as chances de ocorrerem erros por não terem sidos pensados previamente. Para Cornick (1991) e Calavera (1991) apud Tzortzopoulos (1999), esses custos adicionais são provenientes das falhas durante todo o processo de projeto.

Love et al. (2013) evidencia a baixa produtividade da indústria da construção civil nas últimas décadas e aponta a necessidade de abraçar os conceitos de lean construction, métodos de normalização e de pré-montagem e também a utilização de novas tecnologias de modelagem orientada a objetos em três dimensões, para contornar esse quadro. Essas necessidades apontadas pelos autores se encaixam em problemas durante as fases do processo de projeto, como a concepção do empreendimento, técnicas construtivas que serão empregadas e tecnologias que irão auxiliar o processo de desenvolvimento.

Tendo em vista a eclosão do Building Information Modeling (BIM) - Modelagem da Informação da Construção - como novo conceito tecnológico para o processo de projeto, o futuro do trabalho colaborativo será definido através do uso de um modelo único integrado de dados, aos quais todos os agentes envolvidos poderão acessar e editar as informações do projeto em tempo real, desde os estudos preliminares até a fase de operação da construção. Para que seja possível esse avanço na nova forma de se projetar utilizando os conceitos BIM, são necessários grandes esforços pessoais e coletivos por parte dos envolvidos no processo de desenvolvimento do produto, tanto que se diz respeito a cultura colaborativa dos agentes participantes, bem como a sua relação com as tecnologias existentes.

Para Sacks e Melhado (2011), diversas soluções para a ineficácia do processo de projeto de edificações podem ser alcançadas com o uso do BIM, porém o novo patamar de avanço não será alcançado sem que antes sejam resolvidos os problemas existentes na configuração atual da gestão de projetos. Portanto, há o surgimento de uma problemática para a implantação do BIM, de que, da mesma forma que outras tecnologias inseridas no cotidiano da indústria anteriormente, antigas rotinas de trabalho não foram mudadas, passando a utilizar as ferramentas apenas como um meio tecnológico mais moderno para realizar antigas práticas. O presente trabalho buscou julgar o processo de adoção do BIM na cidade de Maceió, desde o conhecimento da utilização das tecnologias no cotidiano das empresas, existência de cargos gerenciais que facilitem a implantação, até a maturidade do conhecimento dos agentes locais sobre o assunto.

\section{COORDENAÇÃO DE PROJETOS E SISTEMAS COLABORATIVOS DE SUPORTE À COORDENAÇÃO DE PROJETOS}

A palavra "coordenação" vem do latim coordinari, que significa "ordenar em conjunto, arranjar, colocar em ordem". O termo apoia a ideia de um arranjo na devida ordem ou na posição própria relativa, cujo objetiva estabelecer relações harmoniosas (harmonizar-se). Ao auxiliar o termo "projetos", obtêm-se uma relação de comando das etapas de projeto, afim 
de organizar os processos para obtenção de um resultado satisfatório para o empreendedor, o construtor e o usuário, considerados clientes do projeto. É de fundamental importância a existência da coordenação entre as atividades de projetos, que compatibilize todas disciplinas, desde os estudos preliminares até o remate e, também, deve realizar $o$ planejamento do projeto visando garantir o fornecimento das informações necessárias à obra.

Caldas e Soilbelman (2001) definem "sistemas colaborativos" como uma rede de computadores que utiliza a tecnologia da Internet para conectar empresas com seus fornecedores, clientes, e outras empresas que compartilham mesmos objetivos, onde é possível haver o controle dos acessos de cada membro do sistema de forma individualizada. Toda a forma de comunicação feita durante a fase de projeto deve ser obrigatoriamente através do sistema colaborativo, podendo ser por e-mails ou transferência de arquivos dentro do próprio sistema. Como estão conectadas a um sistema que gerencia o bando de dados, a informação é processada durante as fases de criação do empreendimento, além de ser armazenada para futuras consultas.

\section{BUILDING INFORMATION MODELING NO PROCESSO DE PROJETO}

Diante do grande volume de dados gerados no processo de projeto, pesquisadores investigaram, durante vários anos, a construção de modelos de produtos virtuais com o auxílio do computador, possibilitando a extração das informações necessárias do produto em qualquer momento que fosse solicitado (SUCCAR, 2009). O BIM, acrônimo de Building Information Modeling, emergiu como o novo Computer-Aided Design (CAD) para dar apoio ao método de desenvolvimento de projetos que busca a colaboração dos profissionais durante todo o ciclo de vida da edificação, através de um modelo virtual compartilhado.

Eastman et al. (2008), define BIM como "uma tecnologia de modelagem e um conjunto de processos associados para produzir, comunicar e analisar modelos de construção". Essa definição propõe um entendimento de que, a partir de tecnologias de modelagem, é possível a produzir modelos de construção virtual para que sejam feitas as diversas análises durante todo o processo de projeto. Para Ayres (2009), entender o BIM como um processo é essencial para a sua efetiva compreensão, já que a modelagem do produto tem como prérequisito básico a integração entre as diferentes fases de desenvolvimento do produto. Então, o foco da ferramenta, em um nível de abstração mais alto, é no apoio a colaboração e cooperação que a ferramenta permite, incluindo a interoperabilidade entre as aplicações, abordagem que é dada neste trabalho.

\subsection{A adesão do BIM}

O início da implementação do BIM no processo de projeto é vagaroso, que envolve a definição de metas de implementação, as ferramentas que serão utilizadas, responsabilidades de cada parte e o escopo da informação a ser compartilhada. Succar (2009) classificou a adoção do BIM em estágios, dependendo de como é feito a colaboração e os propósitos da modelagem, entre outros critérios. Em resumo, os estágios são: Pré-BIM, BIM estágio 1, BIM estágio 2, BIM estágio 3 e BIM estágio - IPD, como mostra a Figura 2. Cada estágio será detalhado mais adiante. 
Figura 2 - Estágios de adoção do BIM segundo Succar (2009)

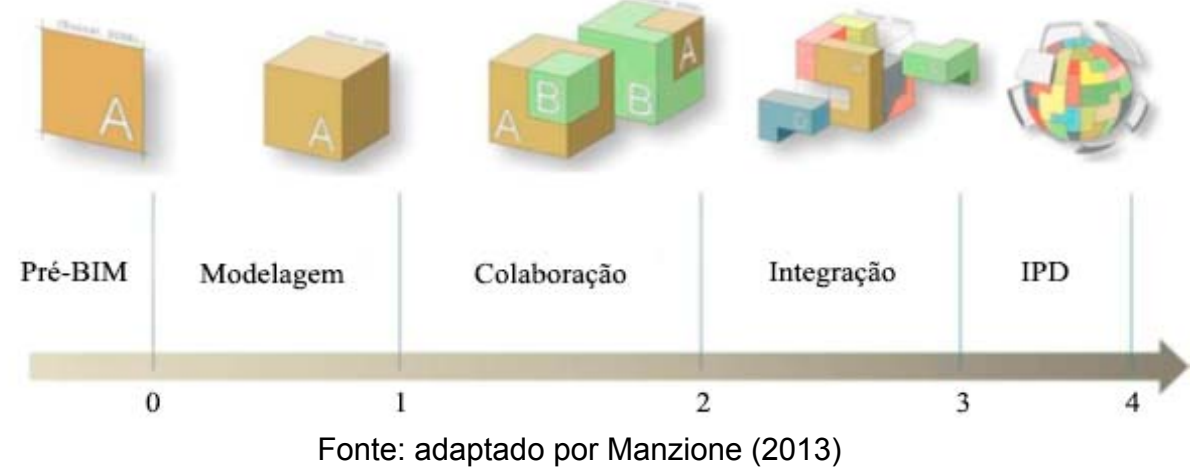

BIM Estágio 0 - (Pré-BIM): Nesse estágio, definido pelo autor como "desarticulado", os desenhos ainda são feitos através de ferramentas CAD 2D com algumas visualizações em 3D, sem integração entre as informações e documentos gerados ao longo do processo de desenvolvimento do projeto pelos distintos intervenientes, como levantamento de quantitativos e especificações.

BIM Estágio 1 - (Modelagem): Segundo o autor, este é o estágio inicial da implementação do BIM através da utilização de algum software paramétrico, 3D e orientado a objetos, como ArchiCAD, Revit, Digital Project, Tekla, entre outros. A modelagem, geralmente, acontece de forma individual entre as disciplinas, para serem utilizas em fases de projeto, construção ou operação. Os modelos comumente gerados nesse estágio são para criação automática de documentação em 2D e visualizações 3D, compatibilização de projetos e tabelas simples de quantitativos de portas, volume de concreto, etc. O processo de trabalho ainda não acontece de forma colaborativa, o intercâmbio de informações entre os agentes é unidirecional e a comunicação se dá de maneira assíncrona e desarticulada.

BIM Estágio 2 - (Colaboração): Após as habilidades adquiridas no estágio anterior quanto a modelagem e utilização de softwares em BIM, o processo colaborativo entre as disciplinas se iniciam através da troca de arquivos em formatos proprietários ou com exportações do formato IFC. A colaboração pode ocorrer em uma ou duas fases do ciclo de vida do edifício como, por exemplo, a troca "projeto-projeto" entre modelos de arquitetura e estrutura, troca "projeto-construção" entre modelo de estrutura e modelos para fabricação de estruturas metálicas e a troca "projeto-operação", entre modelos de arquitetura e modelos de gestão de utilização da edificação.

BIM Estágio 3 - (Integração): Estágio de plenitude para o desenvolvimento do BIM, com modelos semanticamente ricos sendo criados, compartilhados e mantidos de forma colaborativa através do ciclo de vida do projeto. A integração ocorre através do uso de tecnologias como "servidores de modelos" e com utilização de formatos proprietários, abertos ou não proprietários, modelos centrais ou modelos federados e computação na nuvem. Os modelos são interdisciplinares o que favorece as análises mais complexas do desempenho do edifício, desde as primeiras fases do processo de desenvolvimento do produto.

BIM Estágio 4 - (IPD): A colaboração e a absorção do entendimento da tecnologia nesse estágio é o mesmo do estágio anterior, porém, as relações contratuais se alteram, baseando-se na colaboração efetiva por todos os agentes envolvidos, e com a aplicação cos conceitos do IPD - respeito e confiança mútuos; benefícios mútuos e recompensas; inovação e decisões feitas em conjuntos, etc. 


\section{METODOLOGIA DE PESQUISA}

A metodologia utilizada para o desenvolvimento dos resultados deste trabalho caracterizouse pela implantação de uma pesquisa Survey. De acordo com Freitas et al. (2000) a pesquisa Survey pode ser descrita como a obtenção de dados e informações sobre características, ações ou opiniões de determinado grupo de pessoas, indicado como representante de uma população-alvo, por meio de um instrumento de pesquisa, sendo normalmente um questionário.

Figura 3 - Pontos a serem estudados para análise do estágio de implantação do BIM

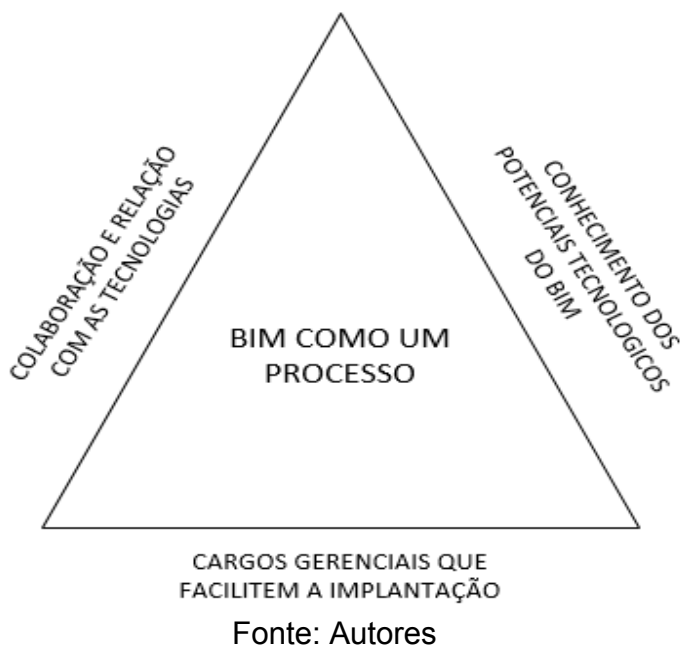

Para que o BIM seja entendido como um processo que tem suas raízes no trabalho colaborativo, é necessário: conhecer as habilidades dos agentes locais em relação ao uso da tecnologia da informação para auxiliar o processo de projeto; estudar a existência de cargos gerenciais que facilitem a implantação e os propósitos para a utilização da tecnologia, que envolve o conhecimento dos entrevistados sobre as ferramentas BIM e quais os benefícios que são alcançados com a sua utilização, conforme ilustrado na Figura 3. É importante compreender essas variáveis para que seja possível avaliar o estágio de adoção atual de acordo com os níveis propostos por Succar (2009), onde a capacidade dos agentes envolvidos no processo é avaliada quanto a utilização das tecnologias e o nível de colaboração alcançada.

Figura 4 - Fluxograma básico para escolha dos profissionais que foram entrevistados

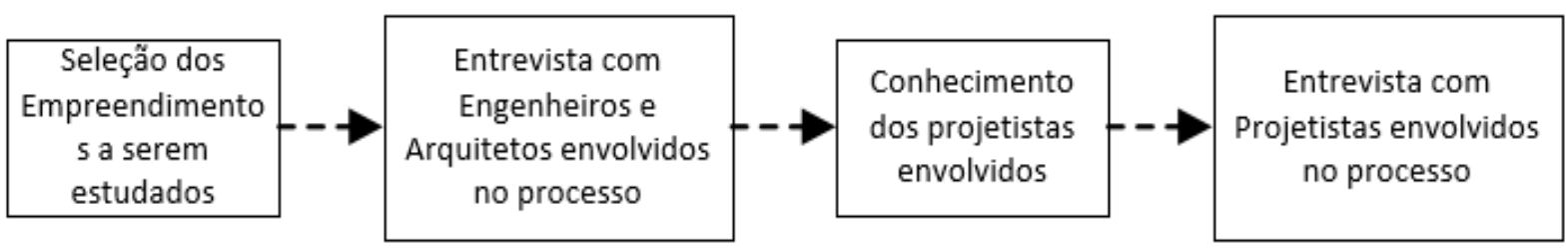

Fonte: Autores

O critério utilizado para seleção dos entrevistados e obtenção de resultados esperados estão representados na Figura 4. Foram feitas entrevistas com as principais empresas atuantes no ramo de construção de edifícios multifamiliares da cidade de Maceió, onde houve a coleta de experiências de engenheiros e arquitetos envolvidos no processo de projeto dos empreendimentos. A partir dessas experiências, foram identificados os principais projetistas envolvidos nesses empreendimentos, pois julgou-se mais adequado 0 conhecimento das opiniões de projetistas que atuam nas obras selecionadas. O critério 
utilizado é demonstrado abaixo, através de um fluxograma. No total, foram coletadas as opiniões de sete empresas que atuam no ramo de construção de empreendimentos de médio e grande porte no âmbito local; dois arquitetos, a qual são os mais solicitados por essas construtoras; dois profissionais de projetos complementares seguindo o mesmo critério anterior e um profissional atuante na área de projetos estruturais.

\section{A UTILIZAÇÃO DE SISTEMAS COLABORATIVOS NO PROCESSO DE PROJETO}

A Figura 5 mostra a situação da utilização dos sistemas colaborativo nas empresas atuantes no ramo de construção de edifício pesquisadas.

Figura 5 - A utilização de sistemas colaborativos no processo de projeto e o tempo de operação

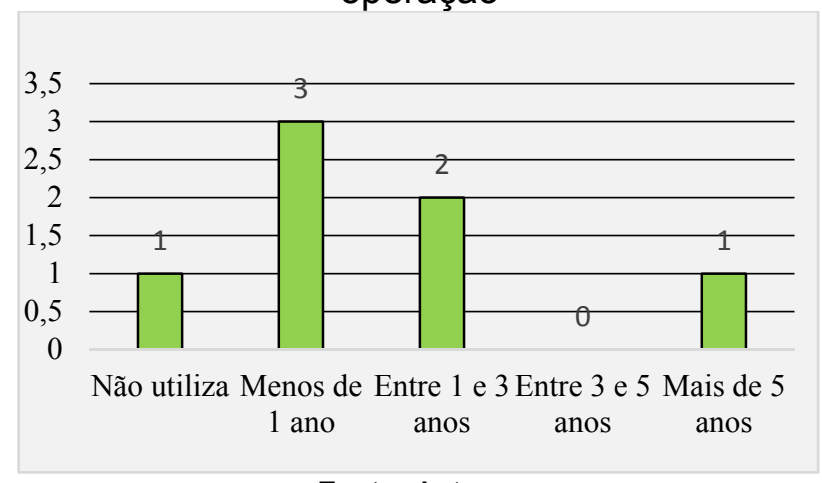

Fonte: Autores

Todos os projetistas participantes do processo de projeto dos empreendimentos selecionados são terceirizados, o que exige uma solução para que as equipes multidisciplinares de projeto trabalhem de forma conjunta. Como resultado do investimento tecnológico em favor da colaboração entre os agentes de projeto, apenas uma empresa não utiliza um sistema colaborativo. A escolha pela utilização dos ambientes de projeto foi motivada pelo grande volume de documentos que estavam sendo gerados, onde haviam muitas informações se perdendo na transição entre as etapas de projeto e construção. $O$ Quadro 2 mostram os benefícios e problemas que não foram solcionados com o uso do sistemas colaborativos..

Quadro 2 - Benefícios e problemas encontrados com asplataformas colaborativas

\begin{tabular}{|c|c|}
\hline $\begin{array}{c}\text { Benefícios encontrados com as plataformas } \\
\text { colaborativas }\end{array}$ & $\begin{array}{c}\text { Problemas encontrados durante o processo de } \\
\text { projeto utilizando as plataformas colaborativas }\end{array}$ \\
\hline $\begin{array}{c}\text { Facilidade de extração de informações devido a } \\
\text { uniformização da mesma }\end{array}$ & Pouca redução nos prazos de projetos \\
\hline Dados precisos concentrados em um único local & Baixas melhorias no processo de projeto e na colaboração \\
\hline $\begin{array}{c}\text { Notifica a todos os envolvidos no processo de } \\
\text { projeto caso haja alguma alteração nos desenhos }\end{array}$ & $\begin{array}{c}\text { Dificuldades na mobilização de todos os projetistas para } \\
\text { utilização da tecnologia }\end{array}$ \\
\hline $\begin{array}{c}\text { Diminuição de projetos obsoletos em obra através do } \\
\text { sistema automático de sobreposição de pranchas } \\
\text { dentro de sistema }\end{array}$ & $\begin{array}{c}\text { Em alguns casos, a comunicação ainda ocorre fora da } \\
\text { plataforma }\end{array}$ \\
\hline Maior facilidade para compatibilizar os projetos & $\begin{array}{c}\text { Alguns métodos para alimentação do sistema são pouco } \\
\text { práticos para os escritórios de projeto }\end{array}$ \\
\hline Aumento do controle sobre as etapas do projeto & $\begin{array}{c}\text { Grande número de construções iniciadas sem os projetos } \\
\text { definitivos }\end{array}$ \\
\hline Melhor administração dos prazos dos projetos & É necessário definir algumas garantias para os projetistas \\
\hline
\end{tabular}




\section{COORDENAÇÃO DO PROCESSO DE PROJETO E A PRESENÇA DO COORDENADOR DE PROJETOS}

A Figura 7 mostra a existência de um coordenador de projetos nas empresas do ramo de construção pesquisadas. Em todos os resultados mostrado em que não há um do coordenador de projetos, é importante destacar a existência de um engenheiro designado a cuidar da produção em obra e que também atua como o coordenador de projetos. No início do processo de concepção do empreendimento, o engenheiro age em conjunto com o empreendedor para auxiliar no estudo de viabilidade econômica da edificação. Após a aprovação do edifício a ser construído, o engenheiro atua como o contratante dos projetistas e estabelece os objetivos do projeto, além do cumprimento de sua função de engenheiro de obra, inserindo seus interesses quanto a execução e a tecnologia construtiva a ser empregada.

Figura 7. Resultado da pesquisa sobre a existência de um coordenador de projetos

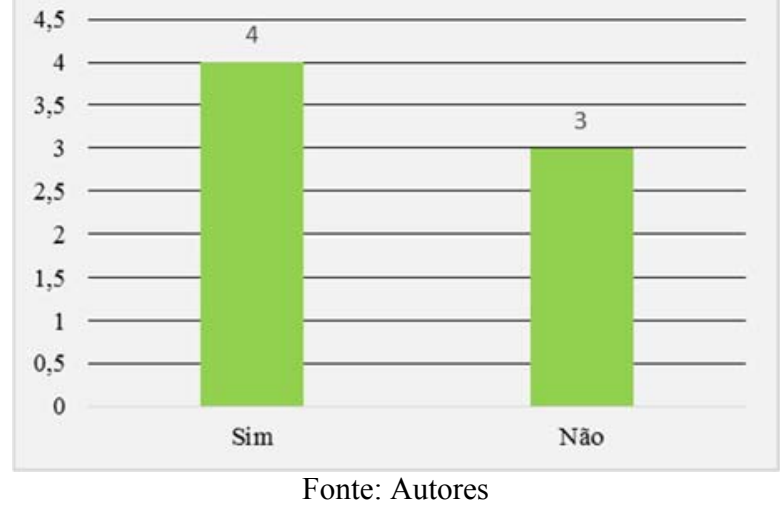

\section{SITUAÇÃO DA IMPLANTAÇÃO DO BIM NO PROCESSO DE PROJETO}

A análise da situação da implantação do BIM consiste, entre outras as coisas, entender a visão dos agentes locais sobre o conceito da modelagem da informação da construção e quais são os benefícios extraídos a partir de sua utilização. A forma de como o BIM é traduzido dentro dos conceitos de um indivíduo, é fundamental para que se obtenha algum grau de expectativa sobre a sua utilização, se será tratada como uma maneira de mudar antigas rotinas ou se serão apenas inseridas no contexto dos métodos de trabalhos convencionais. $O$ entendimento das construtoras e dos projetistas pesquisados sobre 0 termo "BIM" é ilustrado na Figura 8.

Figura 8. O entendimento dos entrevistados sobre o BIM

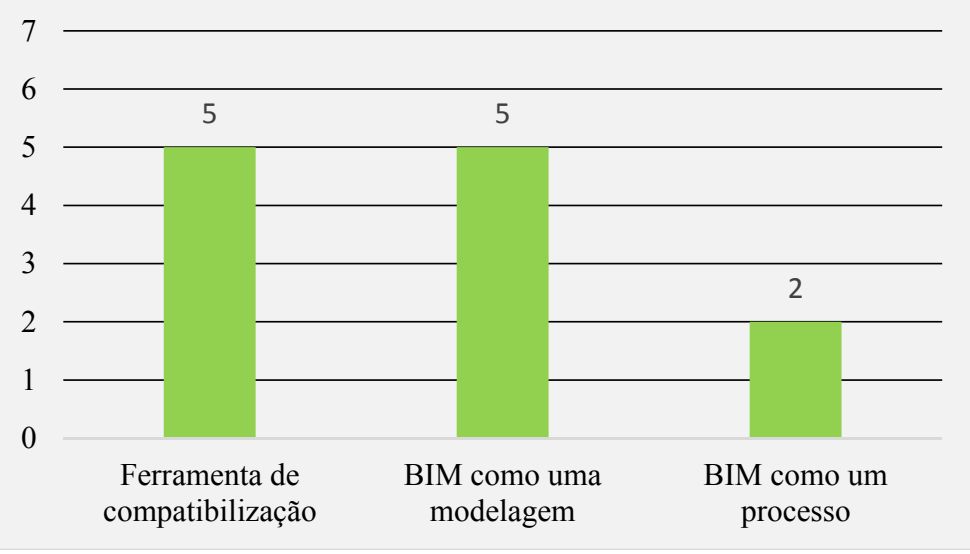

Fonte: Autores 
O marketing excessivo que se criou nos últimos anos em torno do BIM faz com que o termo seja erroneamente associado a um software proprietário ou até mesmo a um benefício que se pode obter com a sua utilização, como no caso da compatibilização de projetos. Decerto, com a associação do termo "BIM" a um benefício proporcionado pela modelagem, é possível entender há o conhecimento sobre alguma determinada ferramenta, porém isso demonstra um desconhecimento sobre o assunto e uma visão bastante limitada sobre a proposta do surgimento do BIM e de sua aplicabilidade.

Associar o BIM a modelagem da informação da edificação, é possível obter uma abordagem mais adequada que a primeira citada, ou menos errônea, uma vez que os estágios de adoção do BIM com propostas colaborativas mais intensas ao longo de todo o processo de projeto deverá se configurar quando os profissionais estiverem com um grau de amadurecimento tecnológico e colaborativo mais consolidado, o que exige tempo. Esse tipo de abordagem para a utilização da tecnologia, com a integração dos conceitos do IPD, ainda é algo muito difícil de se encontrar a nível mundial. Portanto, essa concepção da utilização da ferramenta com foco na modelagem dá margem as várias possibilidades atuais e difundidas para o uso das informações presentes no modelo e garante algumas metas de implantação sejam possíveis de serem planejadas a médio prazo. Enxergar o BIM como um processo traduz um conhecimento mais abrangente de sua utilização, que deve contemplar todo o ciclo de vida de uma edificação através da modelagem da informação.

Sobre a utilização de algum software que trabalha nos conceitos do BIM, apenas duas empresas faz o uso da tecnologia, conforme a Figura 9. Há uma pequena utilização dos softwares BIM nos escritórios de arquitetura pesquisados, mas não foram observados um uso efetivos das ferramentas no desenvolvimento do projeto.

Figura 9. A utilização de algum software BIM no processo de projeto no cenário atual

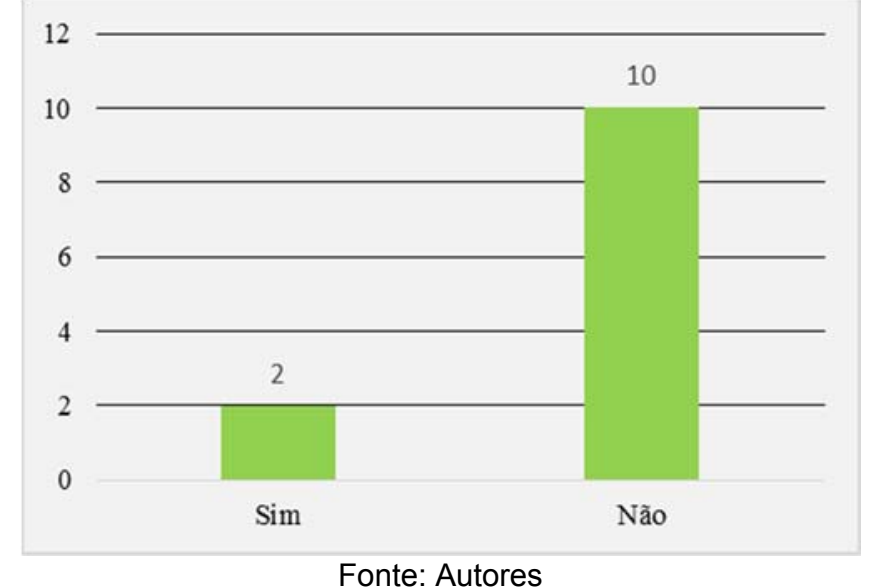

Dentre os principais problemas relatados na pesquisa que impossibilitam a adoção do BIM, destaca-se:

- a atribuição das responsabilidades de implantação aos projetistas;

- impossibilidade de se trabalhar em BIM de forma individual nos estágios iniciais de implantação; elevado custo dos softwares BIM;

- necessidade de treinamento para operação das ferramentas;

- não é viável financeiramente a contratação de profissionais com habilidades com a ferramenta para fazer apenas a modelagem em estágios mais avançados do desenvolvimento de projeto. 
Da mesma forma que outras empresas que iniciaram o processo de implantação do BIM no Brasil, através do entendimento das vantagens obtidas com o uso da modelagem para fins específicos, como: compatibilização de projetos, planejamento de obra, levantamentos de quantitativos e a orçamentação, as empresas pesquisadas atribuíram as mesmas motivações para que se iniciasse o investimento na implantação do BIM. A Figura 10 demonstra as principais motivações das empresas pesquisas com a implantação do BIM.

Figura 10. Principais motivos para a adoção BIM nas empresas pesquisadas

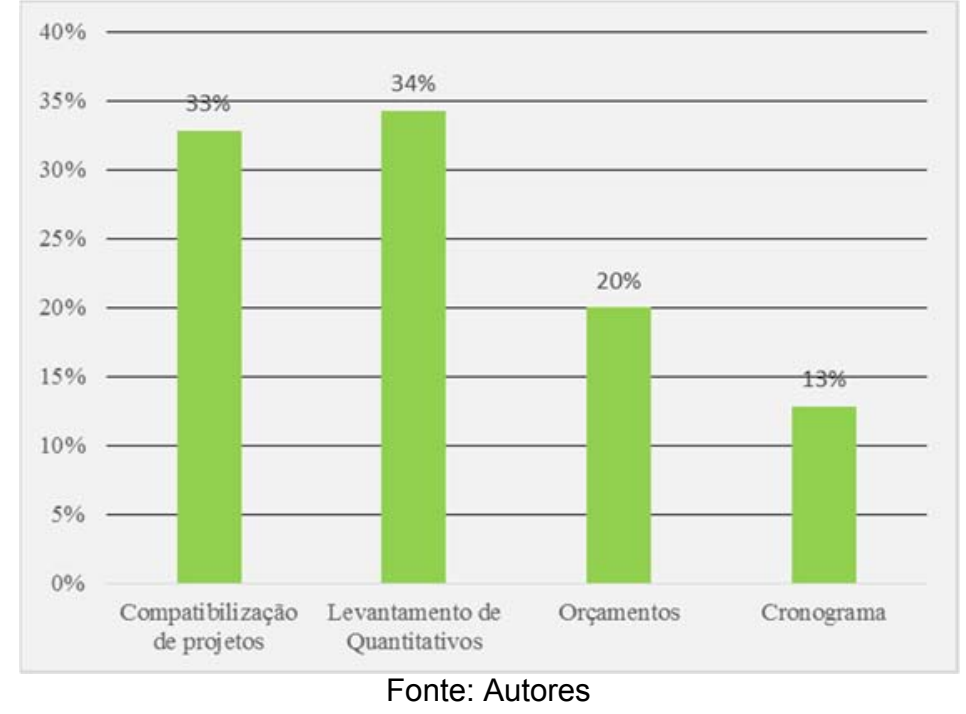

Ao analisar as empresas pesquisadas de acordo com os estágios de adoção propostos por Succar (2009), conforme demonstrado na Figura 11, fica evidente a perspectiva de evolução no processo de implantação do BIM nos próximos anos, porém, ainda se encontra muito aquém do restante do país. É importante destacar que a evolução na utilização do BIM no Estágio 1 para o Estágio 2 necessita um grande desenvolvimento no trabalho colaborativo entre as equipes multidisciplinares de projeto e a obra, estabelecendo um grande desafio para o progresso de evolução no processo de projeto.

É fundamental destacar a importância da presença de um profissional capacitado, com habilidades e conhecimentos sobre gestão do processo de projeto e também sobre gestão da modelagem da informação, para dar continuidade ao processo de evolução do uso do BIM. Dentre as novas formas contratuais que deverão surgir e o estabelecimento de metas para o avanço na utilização da tecnologia, a colaboração ganhará uma dinâmica diferente diante da grande variabilidade de informação que será gerada.

Os esforços se concentrarão no estabelecimento do trabalho colaborativo eficiente, e a modelagem da informação da construção não trará isso diretamente ao processo, assim como as plataformas colaborativas não trouxeram para as empresas pesquisadas. Colaboração não é uma solução tecnológica que pode ser adquirida por meio da tecnologia da informação, é uma solução oriunda das habilidades e da cultura dos profissionais atuantes. 
Figura 11. Inserção dos entrevistados na metodologia de adoção de Succar (2009)

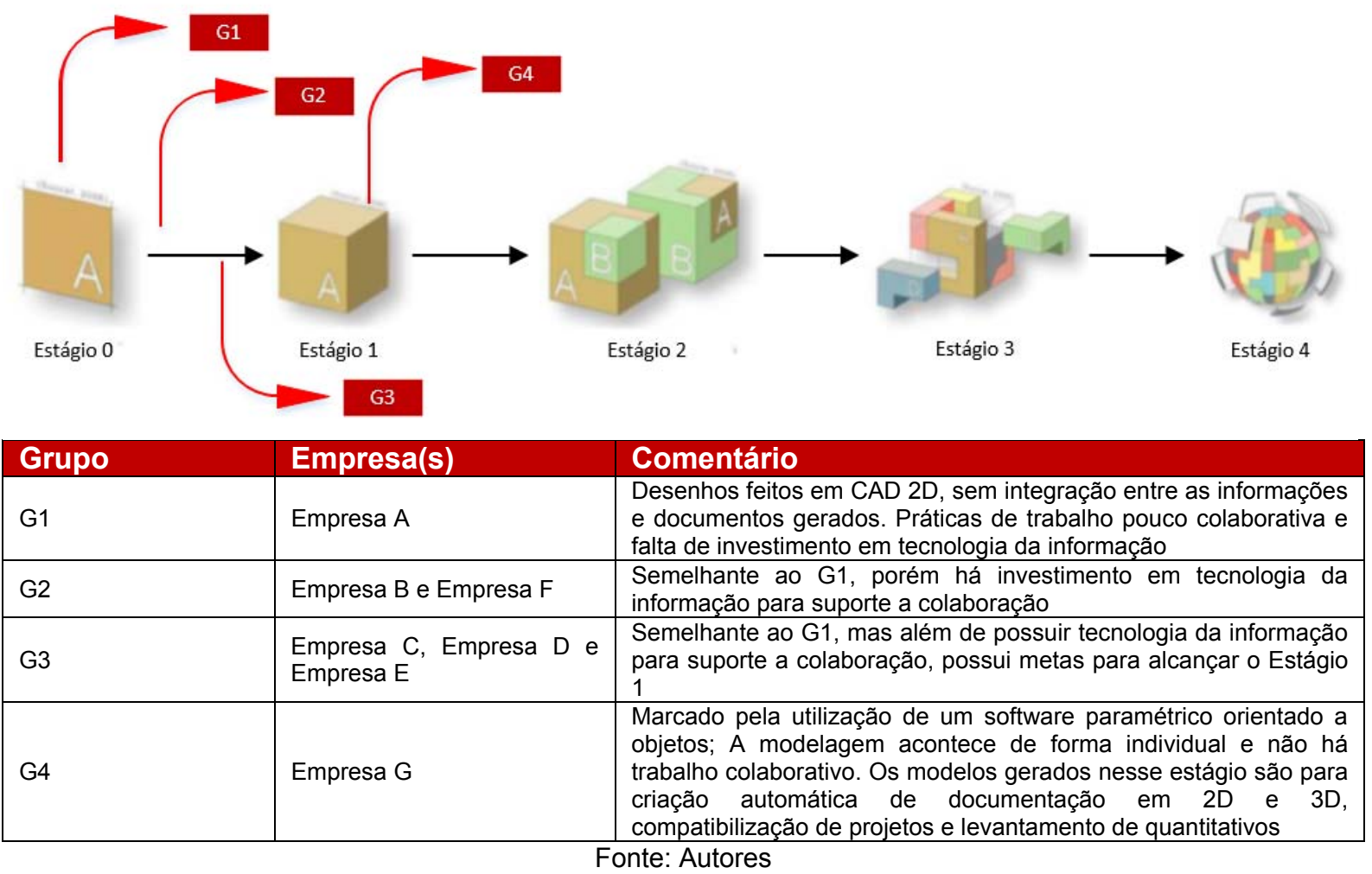

O Quadro 3 mostra alguns dos principais condicionantes apresentados durante a pesquisa de caráter favorável a adoção do BIM.

\begin{tabular}{l} 
Quadro 3. Condicionantes apresentados favoráveis a implantação do \\
$\qquad \begin{array}{r}\text { Condicionantes para a implantação do BIM } \\
\text { Mobilização por parte dos principais projetistas para se inserir em } \\
\text { cursos de treinamento em softwares BIM }\end{array}$ \\
\hline Difusão do termo nos diversos setores da construção civil \\
\hline $\begin{array}{c}\text { Conhecimento das possíveis melhorias alcançadas com a } \\
\text { utilização do BIM }\end{array}$ \\
\hline $\begin{array}{c}\text { A adoção da tecnologia foi caracterizada como definitiva para o } \\
\text { sucesso da empresa nos próximos anos }\end{array}$
\end{tabular}

Fonte: Autores

\section{CONCLUSÕES}

Apesar dos notáveis investimentos em TI, foi possível observar que existem diversos problemas gerenciais a serem resolvidos que impossibilitam que as ferramentas de colaboração exerçam um papel mais significante para a melhoria do processo de projeto nas empresas estudadas. Os sistemas colaborativos não tiveram forte impacto na colaboração 
entre as equipes multidisciplinares de projeto, principal objetivo das ferramentas. A presença da coordenação de projeto, embora existente, foi bastante questionada na pesquisa.

O processo de adoção do BIM envolve o estabelecimento de diversas metas que precisam estar bem definidas para a criação de diretrizes que viabilizam a sua inclusão no processo de projeto e atendam as expectativas dentro da estratégia da empresa. Em estágios iniciais, a pesquisa se mostrou favorável a utilização da ferramenta de forma individual e pouco colaborativa, para que alguns benefícios fossem alcançados mais rapidamente, impulsionando o planejamento de novas metas de utilização em estágios posteriores. Em níveis de propostas mais eficazes, é necessário um estímulo das mudanças culturais na forma de trabalho pouco colaborativa, além do estudo de novas questões contratuais que devem se estabelecer, para que torne possível o nível de fluxo de informações desejado.

Ao analisar o baixo uso efetivo dos sistemas colaborativos, que trouxeram poucas mudanças na colaboração entre as equipes, e as formas de como são coordenados os projetos, sem a valorização do processo por parte dos empreendedores e sem a existência de uma figura que tenha uma participação central durante o desenvolvimento, a proposta de utilização do BIM como um processo precisará de mais alguns anos para amadurecimento. Portanto, o nível de adoção do BIM na cidade estudada pode ser considerado baixo, ou insatisfatório.

\section{REFERÊNCIAS}

AGOPYAN, V. Números do desperdício. Revista Téchne, Ed. 53, ago 2001.

AYRES, C. Acesso ao modelo integrado do edifício. 2009. 254 p. Dissertação (Mestrado) Universidade Federal do Paraná, 2009.

CALDAS, C. H. S.; SOIBELMAN, L. Avaliação da logística de informação em processos interorganizacionais na construção civil. In: Simpósio Brasileiro de Gestão da Qualidade e Organização do Trabalho no Ambiente Construído, $2^{\circ}$, Fortaleza, CE, 2001. Artigo técnico. Porto Alegre. Anais. Porto Alegre: ANTAC, 2001, 11p.

EASTMAN, C.; TEICHOLZ, P.; SACKS, R.; LISTON, K. BIM Handbook: a guide to building information modeling for owners, managers, designers, engineers, and contractors. 2. ed. New Jersey: John Wiley \& Sons, 2011.648p.

FREITAS, H.; OLIVEIRA, M.; SACCOL, A. Z.; MOSCAROLA, J. O método de pesquisa survey. Revista de Administração, São Paulo v.35, n.3, p. 105-112, 2000.

LOVE, P. E.; SIMPSON, I.; HILL, A.; STANDING, C.; From justification to evaluation: Building information modeling for asset owners. Automation in Construction, 35, 208-216, 2013.

SACKS, R.; MELHADO, S. B. Key performance indicators to analyze and improve management of information flow in the BIM design process. Proceedings of the CIB W78-W102 2011: International Conference -Sophia Antipolis, France, 26-28 October, 2011.

MANZIONE, L. Proposição de uma estrutura conceitual de gestão do processo de projeto colaborativo com o uso do BIM. 2013. 389p. Tese (Doutorado) - Escola Politécnica, Universidade de São Paulo, São Paulo, 2013.

SUCCAR, B. Building information modelling framework: A research and delivery foundation for industry stakeholders. Automation in Construction, v. 18, n. 3, p. 357-375, 2009. 
TZORTZOPOULOS, P. Contribuições para o desenvolvimento de um modelo do processo de projeto de edificações em empresas construtoras incorporadoras de pequeno porte. 1999. 150p. Dissertação (Mestrado) - Universidade Federal do Rio Grande do Sul. Porto Alegre. 